Revista Brasileira de Agricultura Irrigada v.7, nº.6, p 340 - 348 , 2013

ISSN 1982-7679 (On-line)

Fortaleza, CE, INOVAGRI - http://www.inovagri.org.br

DOI: $10.7127 /$ rbai.v7n600186

Protocolo 186.13 - 27/09/2013 Aprovado em 03/12/2013

\title{
AVALIAÇÃO DO DESEMPENHO DO MÉTODO DAS PESAGENS PARA A DETERMINAÇÃO DA UMIDADE DO SOLO
}

Taise Cristine Buske ${ }^{1}$, Adroaldo Dias Robaina ${ }^{2}$, Marcia Xavier Peiter ${ }^{3}$, Ricardo Benetti Rosso $^{4}$, Rogério Ricalde Torres ${ }^{5}$, Mario Santos Nunes ${ }^{6}$

\section{RESUMO}

O conhecimento da umidade do solo é de fundamental importância, pois indica em que condições hídricas encontra-se o mesmo. Inúmeros são os métodos utilizados para a determinação, dessa maneira, o presente trabalho objetivou comparar os resultados de umidade do solo obtidos pelo método padrão e pelo método das pesagens para determinação do conteúdo de água no solo em diferentes classes texturais. O estudo foi desenvolvido no Laboratório de Hidráulica Agrícola da Universidade Federal de Santa Maria. Foram testados dois métodos (método padrão de estufa, M0 e método das pesagens, M3), com três amostras de solo (S1, S2, S3) e três repetições para cada. Foi feita a regressão linear entre os métodos, com a finalidade de se obter o coeficiente de correlação ( $r$, o índice de concordância de Wilmott $(I w)$ e o índice de desempenho $\left(I_{d}\right)$. Através dos resultados obtidos pode-se afirmar que o método das pesagens apresentou desempenho bom e muito bom. Também concluiu-se que o método das pesagens se mostrou confiável na determinação da umidade do solo. Portanto, existe a possibilidade de usar o método alternativo para a determinação da umidade do solo.

Palavras-chave: conteúdo de água no solo, comparação de métodos, irrigação

\section{PERFORMANCE EVALUATION OF THE WEIGHING METHOD FOR THE DETERMINATION OF SOIL MOISTURE}

\footnotetext{
${ }^{1}$ Engenheira Agrônoma, Doutoranda Engenharia Agrícola, Universidade Federal de Santa Maria. E-mail: taisecbuske@gmail.com

${ }^{2}$ Engenheiro Agrônomo, Doutor, Professor Titular, Departamento de Engenharia Rural, Universidade Federal de Santa Maria. E-mail: diasrobaina@gmail.com

${ }^{3}$ Engenheira Agrônoma, Doutora, Professora Adjunta, Departamento de Engenharia Rural, Universidade Federal de Santa Maria. E-mail: mpeiter@gmail.com

${ }^{4}$ Engenheiro Agrônomo, Mestrando Engenharia Agrícola, Universidade Federal de Santa Maria. E-mail: cadorosso@gmail.com

${ }^{5}$ Engenheiro Agrônomo, Mestrando Engenharia Agrícola, Universidade Federal de Santa Maria. E-mail: rogeriocprtorres@gmail.com

${ }^{6}$ Engenheiro Agrícola, Doutorando Engenharia Agrícola, Universidade Federal de Santa Maria. E-mail: mario.nunes.s1@gmail.com
} 


\begin{abstract}
The knowledge of soil moisture is quite important because it indicates soil hydric conditions. There are any methods used for determination, in this way, the present study aimed to compare the results of soil moisture obtained by the standard method and by the weighing method for determination of the water content of the soil in different textural classes. The study was conducted in the "Laboratório de Hidráulica Agrícola da Universidade Federal de Santa Maria". Two methods were tested (standard method, M0 and weighing method, M3) with three soil samples (S1, S2, S3) and three repetitions for each. The linear regression was made between the methods, for obtaining the correlation coefficient $(r)$, the Wilmott concordance index $(I w)$ and Performance Index $(I d)$. From the results obtained can be stated that the method of weighing presented a performance good and very good. In addition, it was concluded that the method of weighing was also reliable in determining soil moisture. Therefore, it is possible to use the alternative method to determine the soil moisture.
\end{abstract}

Keywords: water content in the soil, comparison of methods, irrigation

\section{INTRODUÇÃO}

$\mathrm{Na}$ agricultura, a irrigação é fundamental por eliminar o risco de deficiência hídrica nos cultivos, aumentar a produtividade, garantir a produção de alimentos e otimizar o uso do solo. Para uma adequada aplicação de água via irrigação é necessário adotar um adequado manejo, seja ele baseado na estimativa de armazenamento da água no solo, relacionado aos fatores climáticos ou relativos à planta. Segundo Soares et al. (2010), o manejo via solo, que considera a umidade do solo na profundidade efetiva do sistema radicular, é bastante preciso, pois permite determinar a percentagem de água no mesmo.

O monitoramento da umidade do solo possibilita aplicações controladas e pontuais de água, respondendo o quanto e quando irrigar em função das necessidades hídricas das culturas, sendo cada vez mais fundamental na agricultura. Inúmeros são os métodos utilizados para a determinação do conteúdo de água no solo, sendo que para o manejo da irrigação é necessário uma determinação rápida do teor de água no solo.

O método considerado padrão é a extração de água de uma amostra de solo por meio de calor, utilizando-se estufas elétricas, determinando-se, assim, a umidade gravimétrica do solo pela relação entre a massa de água e a massa de solo seco (Klein, 2008). Conforme Santos et al. (2006) é um método destrutivo, direto e muito preciso.

Uma das grandes vantagens do método padrão de estufa é a precisão que ele apresenta, sendo utilizado como referência para calibrar equipamentos e métodos. O maior limitante deste método é o tempo necessário para obtenção do resultado, o qual será de aproximadamente 24 horas. Este método é um dos métodos mais utilizados pelo seu baixo custo de realização, não necessitando de 
equipamentos sofisticados e nem de adição de produtos químicos.

O método das pesagens foi desenvolvido por Papadakis (1941) e melhorado posteriormente por Klar et al. (1966) e Bernardo (1968) apud Righes et al. (2003). O método se baseia na saturação da amostra de solo, contrariamente ao método anterior, que promove o secamento da amostra. É um método simples e, após obtenção do padrão e da densidade de partículas do solo, necessita apenas de balança com sensibilidade de $1 \mathrm{~g}$, sendo, portanto, barato e, para fins práticos apresenta boa precisão (Klar, 1984).

Ghelfi Filho (1988) em seu estudo concluiu que o método das pesagens teve um bom desempenho na determinação da umidade para os três tipos de solos analisados do município de Piracicaba, SP, e a comparação do método das pesagens com o método gravimétrico padrão, mostrou uma alta correlação, independentemente do tipo de solo em questão. Calheiros \& Arndt (1991) descreveram que o método das pesagens possibilitou valores de graus de umidade bem mais próximos daqueles fornecidos pelo secamento em estufa, obtendo, em vários casos, valores idênticos, principalmente no solo de textura argilosa e para graus de umidade mais altos. No solo com textura franco-arenosa ocorreram algumas variações entre os valores de graus de umidade nas subamostras e, de uma maneira geral, foram mais afastados dos fornecidos pelo secamento em estufa, quando comparadas com as de textura argilosa. Os autores ainda afirmam que o método das pesagens pode se constituir num substituto eficiente e preciso do método de secamento em estufa, no controle da aplicação de água de irrigação.

A vantagem deste método é a sua rapidez para a determinação a campo em relação aos demais métodos, pois dá a resposta logo após a retirada da amostra (Bernardo et al., 2006). Além de apresentar um baixo custo e ainda não necessitar de equipamentos sofisticados, podendo ser usado sem restrições para o monitoramento do conteúdo de água no solo para determinar o momento de iniciar a irrigação.

Dessa maneira, o presente trabalho objetiva comparar os resultados de umidade do solo obtidos pelo método padrão e pelo método das pesagens para determinação do conteúdo de água no solo em diferentes classes texturais.

\section{MATERIAL E MÉTODOS}

O presente trabalho foi desenvolvido no Laboratório de Hidráulica Agrícola do Departamento de Engenharia Rural da Universidade Federal de Santa Maria (UFSM). Foram coletadas três amostras de solo (S1, S2 e S3) na camada de 0-0,2 $\mathrm{m}$, de regiões geográficas distintas, ambas no Rio Grande do Sul. O solo S1, localizado no município de Santiago, é classificado como Latossolo Vermelho Distrófico típico, unidade de mapeamento Cruz Alta. O solo S2, de Bossoroca, é classificado como Nitossolo Vermelho Distroférrico latossólico pertencente à unidade de mapeamento São Borja. O solo S3, do município de Alegrete, é 
classificado como Argissolo Vermelho Ditrófico arênico, unidade de mapeamento São Pedro (Streck et al., 2008). Foram escolhidos estes solos devido ao fato de serem possíveis de instalar sistemas de irrigação.

A análise granulométrica foi realizada pelo método da pipeta, a densidade do solo foi determinada de acordo com o método do anel volumétrico, ambos procedimentos descritos em EMBRAPA (1997). A determinação da densidade de partículas foi realizada no Laboratório de Hidráulica da UFSM, pelo método do balão volumétrico modificado (MBVmod) descrito por Gubiani et al. (2006).

Para determinar o teor de umidade dos solos conduziu-se o experimento em estufa com circulação de ar (método padrão, M0), marca CENCO, com dimensões de 0,45 x 0,60 x 0,35 $\mathrm{m}$, e termostato digital TIC-17RGTi. Já no método das pesagens (M3) usou-se um copo de vidro com capacidade de 0,45 litros. Para a pesagem empregou-se uma balança digital com capacidade de $1000 \mathrm{~g}$ e precisão de $0,1 \mathrm{~g}$.

Portanto, foram dois métodos testados (M0, M3), com três amostras de solo (S1, S2, S3) e três repetições para cada, totalizando nove testes para cada método. Sendo as amostras utilizadas para a execução do método padrão de 50 gramas e para as amostras referentes ao método das pesagens foram utilizados $100 \mathrm{~g}$ de cada solo, conforme determina o procedimento experimental. Na sequência acrescentou-se água destilada para valores de umidade gravimétrica de $5,10,15,20$ e $25 \%$, deixando de repouso durante 24 horas. No dia seguinte pesou-se a massa úmida de cada amostra, e em seguida partiu-se para a determinação da umidade do solo pelos métodos propostos neste estudo.

No método padrão de estufa as amostras foram secas durante 24 horas em estufa a 105 ${ }^{\circ} \mathrm{C} \pm 3{ }^{\circ} \mathrm{C}$ e pesadas ao final do procedimento. Estas amostras são pesadas antes e depois da secagem, sendo possível calcular a percentagem de umidade do solo em base seca (Klar, 1984; Righes et al., 2003; Carlesso \& Zimmermann, 2005; Bernardo et al., 2006; EMBRAPA, 1997), conforme a expressão:

$$
U g=\frac{(M u-M s)}{M s \times 100}
$$

Sendo: $U g$ é o conteúdo gravimétrico de água no solo (\% de massa), $M u$ é a massa úmida da amostra (g), e $M s$ é a massa seca da amostra (g).

E no método das pesagens acrescentou-se a massa úmida das amostras água destilada até completar 0,40 litros do copo de vidro. Para a realização deste método é necessário que se calibre o método para cada tipo de solo e profundidade de trabalho, conhecendo assim a massa padrão (Carlesso \& Zimmermann, 2005). Para a obtenção deste padrão adiciona-se água até aproximadamente a metade do volume do copo, em seguida coloca-se $100 \mathrm{~g}$ do solo seco em estufa a $105^{\circ} \mathrm{C}$ agitando bem para garantir uma boa homogeneização da mistura água-solo. Posteriormente, completa-se o volume do frasco com água, e pesa-se o conjunto $(M)$, considerado como padrão para o solo em questão, o qual é determinado apenas uma vez.

Para a determinação do conteúdo de água, repete-se, com a amostra de solo que se quer 
determinar a umidade, os mesmos passos utilizados para a obtenção do padrão, exceto a secagem do solo, obtendo-se o peso $M^{\prime}$. O cálculo da umidade com base em peso úmido $(U$, em \%) é efetuado por meio da equação abaixo (Righes et al., 2003):

$$
U=\left(M-M^{I}\right) \times\left(\frac{D p}{D p-1}\right)
$$

Onde: $D p=$ densidade de partículas do solo, geralmente, igual a 2,65 g.cm ${ }^{-3}$.

Para a obtenção do conteúdo de água no solo em \% de massa (em base seca) pode ser utilizada a seguinte equação:

$$
U g=100 \times \frac{U}{100-U}
$$

Onde: $U g$ é o conteúdo de água no solo em relação à massa seca (\%), e $U$ é o conteúdo de água no solo em relação à massa úmida $(\%)$.

Executou-se a regressão linear $Y=a+b X$ entre o método alternativo $(X)$ e o método padrão de estufa ( $Y$ ), pelo software SigmaPlot 11.0, com a finalidade de se obter o coeficiente de correlação $(r)$, o coeficiente linear e o coeficiente angular, e o índice de concordância de Wilmott (Iw). Após a obtenção dos valores dos coeficientes $(a, b)$ da regressão linear foi feito o teste $t$ de Student, ao nível de confiança de $95 \%$.

O coeficiente de correlação permite quantificar o grau de associação entre as duas variáveis envolvidas na análise (Schneider et al., 1998). O campo de variação é de -1 a 1 e quanto maior o seu valor absoluto maior o grau de associação. $\mathrm{O}$ coeficiente de correlação pode ser estimado por:

$$
r=\frac{\sum_{i=1}^{n}\left(E_{i}-\bar{E}\right) \times\left(O_{i}-\bar{O}\right)}{\sqrt{\left[\sum_{i=1}^{n}\left(E_{i}-\bar{E}\right)^{2}\right]\left[\sum_{i=1}^{n}\left(O_{i}-\bar{O}\right)^{2}\right]}}
$$

Onde: $E_{i}$ são os valores estimados, $\mathrm{O}_{\mathrm{i}}$ os valores observados, $\bar{E}$ a média dos valores estimados e $\bar{O}$ a média dos valores observados.

O índice de concordância fornece o grau de exatidão entre as variáveis envolvidas, uma vez que está relacionada a diferença entre os valores estimados em relação aos valores observados, sendo seu campo de variação de 0 (nenhuma concordância) a 1 (concordância perfeita) (Willmott, 1981). O índice de concordância de Willmott é calculado por:

$$
I W=1-\left[\frac{\sum_{i=1}^{n}\left(E_{i}-O_{i}\right)^{2}}{\sum_{i=1}^{n}\left(\left|E_{i}-\bar{E}\|+\| O_{i}-\bar{O}\right|\right)^{2}}\right]
$$

Onde: $\mathrm{E}_{\mathrm{i}}$ é o valor da observação estimada, $\mathrm{O}_{\mathrm{i}}$ é o valor da observação medida e $\bar{E}$ é a média dos valores da observação estimada e $\bar{O}$ é a média da observação medida.

A avaliação do desempenho das fórmulas utilizadas foi feita pelo índice de desempenho $\left(I_{d}\right)$, proposto por Camargo \& Sentelhas (1997), cujo valor é o produto do coeficiente de correlação e o índice de concordância $(I d=r \times I w)$. O critério de interpretação do índice de desempenho e das respectivas classes 
de desempenho utilizadas neste trabalho pode ser visto na Tabela 1.

Tabela 1. Critério de interpretação dos valores do índice desempenho e as respectivas classes de desempenho.

Critério de interpretação do índice de desempenho

Classes de desempenho

\begin{tabular}{|c|c|c|c|c|c|}
\hline 0,85 & $<$ & Id & & & Ótimo \\
\hline 0,76 & $<$ & Id & $\leq$ & 0,85 & Muito Bom \\
\hline 0,66 & $<$ & Id & $\leq$ & 0,76 & Bom \\
\hline 0,61 & $<$ & Id & $\leq$ & 0,66 & Mediano \\
\hline 0,51 & $<$ & Id & $\leq$ & 0,61 & Ruim \\
\hline \multirow[t]{2}{*}{0,41} & $<$ & Id & $\leq$ & 0,51 & Muito Ruim \\
\hline & & Id & $\leq$ & 0,41 & Péssimo \\
\hline
\end{tabular}

\section{RESULTADOS E DISCUSSÃO}

A análise granulométrica foi realizada com a finalidade de caracterizar o solo local, e os resultados obtidos das características físicas dos três solos estudados são apresentados na tabela 2.

Tabela 2. Características físicas dos solos estudados.

\begin{tabular}{cccccc} 
& Profundidade & \multicolumn{3}{c}{ Granulometria (\%) } & \\
\cline { 3 - 5 } Amostra & & & & \\
\cline { 3 - 5 } & $(\mathrm{m})$ & Areia & Silte & Argila & \\
\hline S1 & $0-0,2$ & 45,4 & 18,7 & 35,9 & Argilo arenoso textural \\
S2 & $0-0,2$ & 6,2 & 30,2 & 63,6 & Muito argiloso \\
S3 & $0-0,2$ & 70,3 & 2,4 & 27,3 & Franco argilo arenoso
\end{tabular}

Conforme os resultados obtidos pode-se perceber que são solos com classes texturais distintas, portanto, justificando o comportamento diferenciado frente a propriedades físicas como a capacidade de retenção de água. Segundo Michelon et al. (2007) os solos de áreas irrigadas da região das missões e do planalto do Estado do Rio Grande do Sul apresentam teores médios de argila na faixa de 400 a $500 \mathrm{~g} \mathrm{Kg}^{-1}$ na camada superficial.

O solo S1 apresentou densidade média do solo de $1,55 \mathrm{~g} \mathrm{~cm}^{-3}$ e densidade média de partículas de $2,56 \mathrm{~g} \mathrm{~cm}^{-3}$. O solo $\mathrm{S} 2$, densidade média do solo de $1,8 \mathrm{~g} \mathrm{~cm}^{-3}$ e densidade média de partículas de 2,43 $\mathrm{g} \mathrm{cm}^{-3}$. No solo $\mathrm{S} 3$ foi encontrada densidade média do solo de 1,7 $\mathrm{g}$ $\mathrm{cm}^{-3}$ e densidade média de partículas de 2,61 $\mathrm{g}$ $\mathrm{cm}^{-3}$. Os solos aqui estudados tem um histórico de intenso uso agrícola, determinando a elevada densidade do solo. Os valores da densidade de partícula observados pertencem a faixa de variação de 2,3 a 3,0 $\mathrm{g} \mathrm{cm}^{-3}$ descrita por Kaiser (2010).

A comparação gráfica dos valores de umidade gravimétrica do método da estufa com o método das pesagens, nos percentuais de umidade estabelecidos é apresentada na Figura 1, para os três solos estudados. Para ambos os gráficos apresentados foi ajustada uma equação linear, com valores para y, o método padrão, em relação ao método das pesagens (x). Sendo que, as equações apresentaram coeficientes de determinção de $0,76,0,78$ e 0,81 para o $S 1$, S2 e S3, respectivamente, indicando haver um ajustamento aceitável dos valores de umidade. 

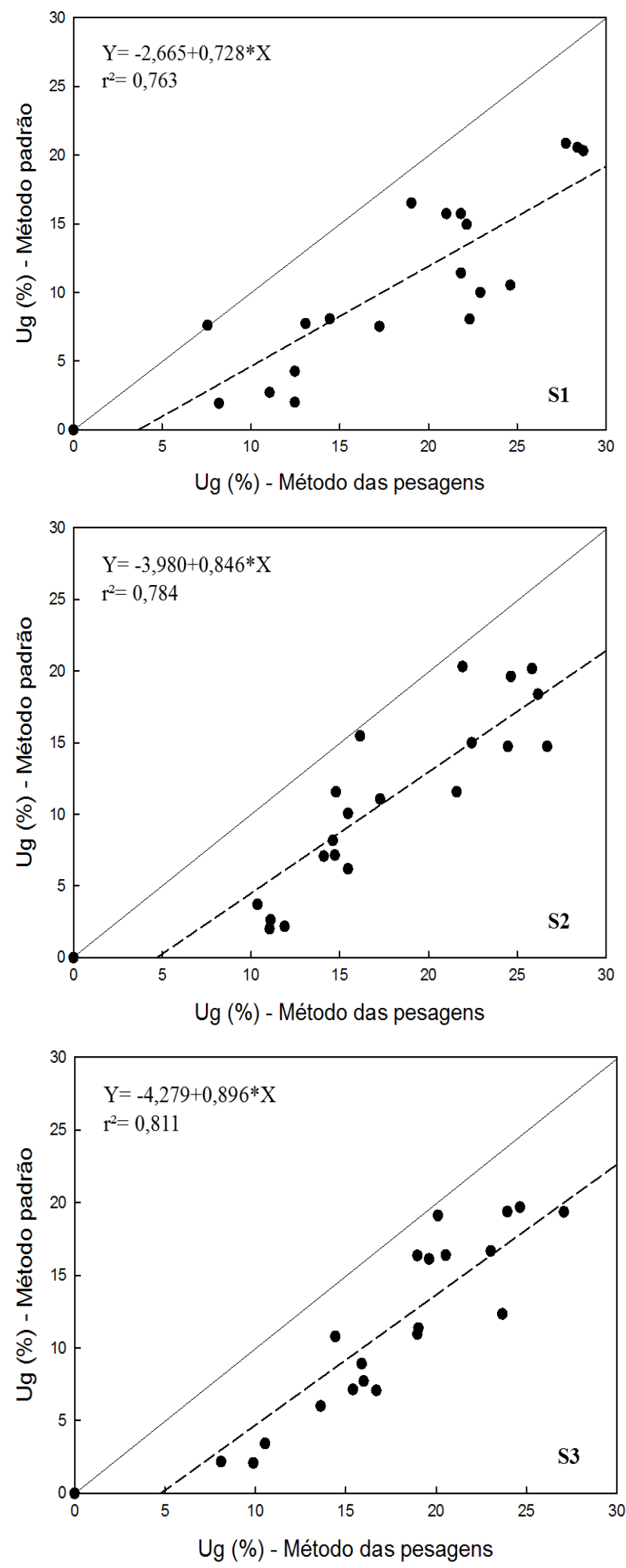

Figura 1. Comparação entre os valores de umidade gravimétrica (Ug) obtidos pelo método da estufa e o método das pesagens para o S1, S2 e S3, respectivamente.
Analisando as equações encontradas podese afirmar que os valores do coeficiente angular foram não significativos ao nível de $5 \%$ de probabilidade de erro, indicando que $b=1$. Resultado este percebido tanto quando se avalia todos os solos reunidos, assim como, quando se avalia cada solo isoladamente. Portanto, pode-se afirmar que o médodo das pesagens é um método confiável para a determinação da umidade do solo, visto que não diferiu estatisticamente do método padrão de estufa. De forma que existe a possibilidade de usar este método para determinar a umidade do solo em substituição ao método padrão.

Resultados semelhantes foram observados por Filho (1988) onde concluiu que o método das pesagens teve um bom desempenho na determinação da umidade para os três tipos de solos analisados (texturas argilosa, barro arenosa e barro argilosa), e a comparação do método das pesagens com o método gravimétrico padrão, mostrou uma alta correlação, independente do tipo de solo em questão. Este autor ainda relata a facilidade de execução do método, visto que com apenas um frasco e uma pequena balança obteve-se resultados confiáveis.

Os valores do coeficiente de correlação $(r)$, índice de concordância $(I w)$, índice de desempenho $(I d)$ e classe de desempenho para o método analisado neste trabalho é apresentado na Tabela 3. 
Tabela 3. Valores do coeficiente de correlação, índice de concordância, índice de desempenho e classe de desempenho para os métodos estudados.

\begin{tabular}{ccccc} 
Tratamentos & $r$ & Iw & Id & $\begin{array}{c}\text { Classe de } \\
\text { desempenho }\end{array}$ \\
\hline M3 S1 & 0,860 & 0,839 & 0,722 & Bom \\
M3 S2 & 0,885 & 0,884 & 0,783 & Muito bom \\
M3 S3 & 0,906 & 0,909 & 0,824 & Muito bom
\end{tabular}

Como pode ser observado no quadro acima, o coeficiente de correlação indicou boa associação entre as variáveis envolvidas na análise. Em relação ao índice de concordância, que fornece o grau de exatidão entre as variáveis envolvidas, pode-se observar que a concordância é quase perfeita. Ainda percebe-se que os desempenhos variaram entre bom $\mathrm{e}$ muito bom. As prováveis razões para estes resultados se devem a dificuldade na leitura do nível estabelecido no copo. Pois ao misturar a água destilada à massa de solo forma-se espuma, que pode vir a confundir na leitura, afetando diretamente nos valores encontrados de umidade gravimétrica.

Por estes resultados pode-se afirmar que a metodologia aplicada apresentou resultados consistentes, podendo ser esta uma alternativa para estimativa da umidade referente ao método padrão de estufa. $\mathrm{O}$ método das pesagens apresenta-se como um método com grande potencial de utilização pelos produtores e técnicos para se medir a umidade do solo, visto que é simples e rápido. Além de apresentar um baixo custo e ainda não necessitar de equipamentos sofisticados, podendo ser utilizado sem restrições para o monitoramento do conteúdo de água no solo para determinar o momento de iniciar a irrigação.

\section{CONCLUSÕES}

Segundo a metodologia utilizada neste estudo e os resultados obtidos, foi possível concluir que as amostras de solo apresentaram características físicas distintas. O método das pesagens apresentou desempenho bom e muito bom quando comparado ao método padrão de estufa, podendo ser um método confiável na determinação da umidade do solo, tendo como principais vantagens o baixo custo e a rapidez na determinação.

\section{REFERÊNCIAS BIBLIOGRÁFICAS}

BERNARDO, S.; SOARES, A. A.; MANTOVANI, E. C. Manual de irrigação. 8.ed. - Viçosa: Ed. UFV, 2006. 625p.

CALHEIROS, C. B. M.; ARNDT, E. Comparação de métodos de determinação da umidade do solo para fins de irrigação. Ciência Agrícola, [S.1.], v. 1, n. 1, p. 7-14, 1991.

CAMARGO, A. P. de; SENTELHAS, P.C. Avaliação do desempenho de diferentes métodos de estimativa da evapotranspiração potencial no Estado de São Paulo, Brasil. Revista Brasileira de Agrometeorologia, Santa Maria, v.5, p.89-97, 1997.

CARLESSO, R.; ZIMMERMANN, F. L. Água no solo: Parâmetros para dimensionamento de sistemas de irrigação. Santa Maria: UFSM/ Departamento de Engenharia Rural, 2000. 88p. (Caderno Didático ${ }^{\circ} 4$ ). 
EMBRAPA. Centro Nacional de Pesquisa de Solos (Rio de Janeiro, RJ). Manual de métodos de análise de solo / Centro Nacional de Pesquisas de Solos. - 2. ed. rev. atual. - Rio de Janeiro, 1997. 212p.

GHELFI FILHO, H. Estudo comparativo do método das pesagens e o método gravimétrico padrão para a determinação do teor de umidade do solo. Anais... ESALQ, Piracicaba, 1988.

GUBIANI, P. I.; REINERT, D. J.; REICHERT, J. M. Método alternativo para a determinação da densidade de partículas do solo - exatidão, precisão e tempo de processamento. Ciência Rural, Santa Maria, v.36, n. 2, p. 664-668, mar./abr. 2006.

KAISER, D.R. Universidade Federal de Santa Maria. Centro de Ciências Rurais. Fundamentos da ciência do solo. Aulas práticas. Santa Maria, 2010. 112 p.

KLAR, A. E. et al. Determinação da umidade do solo pelo método das pesagens. In: Anais da Escola Superior de Agricultura Luis de Queiroz, 1966, Piracicaba. Anais... Piracicaba, v. 23, p. 15-30, 1966.

KLAR, A. E. A água no sistema solo-plantaatmosfera. São Paulo: Nobel, 1984. 408p.

KLEIN, V. A. Física do Solo - Passo Fundo:
Ed. Universidade de Passo Fundo, 2008. 212p.

MICHELON, C. J. et al. Qualidade física de solos irrigados do Estado do Rio Grande do Sul. Ciência Rural, Santa Maria, v.37, n.5, p. 13081315, set./out. 2007.

RIGHES, A. A. et al. Determinação da água no solo e na planta para irrigação. Santa Maria: Imprensa Universitária, 2003. 97p.

SANTOS, R. M. et al. Montagem e acurácia de um sistema experimental de pesagem para calibração de sensores de umidade de solo. Ciência e agrotecnologia, Lavras, v. 30, n. 6, p. 1162-1169, nov./dez. 2006.

SCHNEIDER, P. R. Análise de regressão aplicada à Engenharia Florestal. Santa Maria: UFSM/CEPEF, 1998. 236p.

SOARES, F. C. et al. Resposta da produtividade de híbridos de milho cultivado em diferentes estratégias de irrigação. Irriga, Botucatu, v. 15, n. 1, p. 36-50, jan./mar. 2010.

STRECK, E. V. et al. Solos do Rio Grande do Sul. 2.ed. Porto Alegre: EMATER/ RS, 2008. 222p.

WILLMOTT, C. J.: On the validation of models. Physical Geography, v.2, n. 2, p.184194, 1981. 